\title{
The HMGB1-IL-17A axis contributes to hypoxia/reoxygenation injury via regulation of cardiomyocyte apoptosis and autophagy
}

\author{
XIAORONG HU ${ }^{1}$, KAI ZHANG ${ }^{2}$, ZHIQIANG $\mathrm{CHEN}^{2}$, HONG JIANG ${ }^{1}$ and WEIPAN XU ${ }^{2}$ \\ ${ }^{1}$ Department of Cardiology, Renmin Hospital of Wuhan University, Cardiovascular Research Institute of \\ Wuhan University, Wuhan, Hubei 430060; ${ }^{2}$ Department of Cardiology, Huangshi Central Hospital, Affiliated \\ Hospital of Hubei Polytechnic University, Edong Healthcare Group, Huangshi, Hubei 435000, P.R. China
}

Received April 19, 2016; Accepted March 17, 2017

DOI: $10.3892 / \mathrm{mmr} .2017 .7839$

\begin{abstract}
Both the high-mobility group box 1 protein(HMGB1) and interleukin (IL)-17A serve important roles in myocardial ischemia and reperfusion injury. The purpose of the present study was to evaluate whether HMGB1 could induce IL-17A secretion and lead to cardiomyocyte hypoxia/reoxygenation $(\mathrm{H} / \mathrm{R})$ injury. Neonatal rat cardiomyocytes were treated with HMGB1-neutralizing antibody, IL-17A-neutralizing antibody, recombinant HMGB1 (rHMGB1) and recombinant IL-17A (rIL-17A), respectively. Cell viabilities, lactate dehydrogenase and creatine kinase levels were measured. Apoptotic cells were assessed by flow cytometry. The expression of HMGB1, IL-17A, microtubule-associated proteins 1A/1B light chain 3B (LC3), Beclin-1, B-cell lymphoma (Bcl)-2 and Bcl-2-associated $\mathrm{X}$ protein were assessed by western blot analysis. The results demonstrated that HMGB1 significantly increased the expression of IL-17A. HMGB1 or IL-17A antibody significantly ameliorated H/R-induced cell injury and improved the cell viability. In contrast, rHMGB1 or rIL-17A aggravated cell injury and inhibited the cell viability. Furthermore, cardiomyocytes were treated with HMGB1 or IL-17A antibody significantly increased $\mathrm{Bcl}-2$ protein expression and had fewer apoptotic cells, whereas rHMGB1 or rIL-17A-treated cardiomyocytes markedly decreased $\mathrm{Bcl}-2$ protein expression and had more apoptotic cells. Moreover, HMGB1 or IL-17A antibodies significantly inhibited H/R induced autophagy dysfunction (as determined by the inhibition of Beclin-1 expression, a lower ratio of LC3-II to LC3-I), whereas rHMGB1 or rIL-17A may promote cardiomyocyte autophagy. Together, these results suggested that the HMGB1-IL-17A axis
\end{abstract}

Correspondence to: Dr Weipan Xu, Department of Cardiology, Huangshi Central Hospital, Affiliated Hospital of Hubei Polytechnic University, Edong Healthcare Group, 141 Tianjin Road, Huangshi, Hubei 435000, P.R. China

E-mail: xuweipan@whu.edu.cn

Key words: high-mobility group box 1 protein, interleukin-17A, apoptosis, autophagy, myocardial hypoxia and reoxygenation contributes to $H / R$ injury via regulation of cardiomyocyte apoptosis and autophagy.

\section{Introduction}

Myocardial ischemia/reperfusion (I/R) injury is a complex pathophysiological process, including an inflammatory response, apoptotic cell death and autophagy, which may result in myocardial cell damage (1-3). Therefore, prevention and treatment of myocardial I/R injury has become one of the most important tasks for patients with ischemic heart disease.

The interleukin (IL)-17 cytokine family consists of six members (IL-17A-F). IL-17A and IL-17F share the greatest similarity, with 55\% homology at the amino acid level. IL-17A is a member of the IL-17 cytokine family, secreted by $\mathrm{CD} 4^{+} \alpha \beta$ $\mathrm{T}$ cells, rठT cells, natural killer cells and neutrophils (4). In myocardial I/R injury, it has been indicated that IL-17A is produced primarily by roT cells and plays a pathogenic role by inducing cardiomyocyte apoptosis and neutrophil infiltration (5). These indicated that IL-17A serves a critical role in myocardial I/R injury.

High-mobility group box 1 protein (HMGB1), a highly conserved nuclear protein that is released from necrotic cells and secreted by activated macrophages, natural killer cells and mature dendritic cells, can function as an extracellular signaling molecule during inflammation, cell differentiation, cell migration and tumor metastasis (6). Andrassy et al (7) demonstrated that the expression of HMGB1 was significantly increased during the reperfusion period and could exaggerate myocardial I/R injury, while inhibiting HMGB1 could reduce myocardial I/R injury. A recent study indicated that HMGB1 could aggravate the cerebral I/R injury by upregulating the expression of IL-17A, as well as increasing neuronal apoptosis (8). In addition, a previous study of the authors reported that HMGB1-IL-17A axis serves an important role in the pathogenesis of myocardial I/R injury (9). However, whether the HMGB1-IL-17A axis is involved in regulating cardiomyocyte apoptosis and autophagy in myocardial I/R injury remains unclear. The current study, therefore, aims to investigate the HMGB1-IL-17A axis in a cardiomyocyte hypoxia/reoxygenation (H/R) injury model to elucidate its possible role in regulating cardiomyocyte apoptosis and autophagy in myocardial I/R injury. 


\section{Materials and methods}

Cell culture and experimental design. The cell extraction protocol for cardiomyocyte primary culture was approved by the Institutional Animal Care and Use Committee of Wuhan University (Wuhan, China). A total of 100 Sprague-Dawley rats (1-3-day-old) were purchased from the Centre of Experimental Animals at Wuhan University (Wuhan, China). Primary cultures of neonatal rat cardiomyocytes were prepared from the ventricles of rats. Briefly, the hearts were harvested and finely minced with scissors for about $1 \mathrm{~min}$ and then dissociated with $0.125 \%(\mathrm{w} / \mathrm{v})$ trypsin and $0.08 \%$ collagenase I for five times at $37^{\circ} \mathrm{C}$. The collected enzyme solution is next centrifuged $\left(170 \mathrm{x} \mathrm{g}, 4 \mathrm{~min}, 4^{\circ} \mathrm{C}\right)$, the supernatant discarded, and cells re-suspended in Dulbecco's modified Eagle's medium (DMEM, Sigma-Aldrich; Merck KGaA, Darmstadt, Germany) containing $15 \%(\mathrm{v} / \mathrm{v})$ fetal bovine serum (FBS), $1 \%$ penicillin $(100 \mathrm{U} / \mathrm{ml})$ and $1 \%$ streptomycin $(100 \mu \mathrm{g} / \mathrm{ml})$. To reduce fibroblast contamination, cells were incubated at $37^{\circ} \mathrm{C}$ and $5 \% \mathrm{CO}_{2}$ for $1 \mathrm{~h}$, then non-adherent cells (cardiomyocytes) in DMEM were collected and seeded in six-well plates at a density of $1 \times 10^{6}$ cells $/ \mathrm{ml}$. Cardiomyocytes were incubated for 4 days prior to conducting the experiment, then the cells were randomly assigned into six groups. The group descriptions are as follows: Control group (Control), cardiomyocytes were incubated in DMEM F12 with 15\% FBS; Hypoxia and reoxygenation (H/R) group, cardiomyocytes were incubated in DMEM following $24 \mathrm{~h}$ of synchronization, subjected to hypoxia for $1 \mathrm{~h}$ at $37^{\circ} \mathrm{C}$ and $95 \% \mathrm{~N}_{2}$ at $5 \%(\mathrm{v} / \mathrm{v}) \mathrm{CO}_{2}$ prior to incubation in DMEM F12 for $4 \mathrm{~h} ; \mathrm{H} / \mathrm{R}+$ anti-HMGB1 group, cardiomyocytes were pretreated with HMGB1-neutralizing antibody (1:100 dilution; \#BM3965; Wuhan Boster Bioengineering Co, Ltd., Wuhan, China) at $37^{\circ} \mathrm{C}$ for $24 \mathrm{~h}$ before incubation in DMEM, and then were subjected to $\mathrm{H} / \mathrm{R}$, as described above; H/R+anti-IL-17A group, cardiomyocytes were pretreated with IL-17A-neutralizing antibody (1:100 dilution; \#A00421; Wuhan Boster Bioengineering Co, Ltd.) at $37^{\circ} \mathrm{C}$ for $24 \mathrm{~h}$ before incubation in DMEM, and then were subjected to $\mathrm{H} / \mathrm{R}$, as described above; $\mathrm{H} / \mathrm{R}+\mathrm{rHMGB} 1$ group, cardiomyocytes were pretreated with recombinant HMGB1 (rHMGB1; $200 \mathrm{ng} / \mathrm{ml}$ ) at $24 \mathrm{~h}$ before incubation in DMEM, and then were subjected to $\mathrm{H} / \mathrm{R}$, as described above; and the H/R+rIL-17A group, cardiomyocytes were pretreated with recombinant IL-17A (rIL-17A; $100 \mathrm{ng} / \mathrm{ml}$ ) at $24 \mathrm{~h}$ before incubation in DMEM, and then were subjected to $H / R$, as described above.

Assessment of cell injury. The extent of cell injury was assessed by the concentrations of lactate dehydrogenase (LDH) and creatine kinase $(\mathrm{CK})$ in the culture medium. The protocols were followed according to the manufacturer's instructions (Nanjing Jiancheng Bioengineering Institute, Nanjing, China).

Assay of cell viability. Cell viability was determined using the Cell Counting Kit (CCK)-8 assay (Dojindo Molecular Technologies, Inc., Kumamoto, Japan), and the experimental procedure was based on the manufacturer's recommendations. Cardiomyocytes were seeded in 96-well plates at $1 \times 10^{5}$ cells/well and incubated for $4 \mathrm{~d}$ prior to treatments as described above. The absorbance of each well at $490 \mathrm{~nm}$ was measured with a microplate reader (Bio-Rad Laboratories,
Hercules, CA). The percent cell viability was calculated using the following formula: \% cell viability=(mean absorbance in test wells)/(mean absorbance in control wells) x100.

Flow cytometry. Apoptosis rate was assessed by flow cytometric analysis of propidium iodide (PI) and Annexin V double staining. The cardiomyocytes were harvested after treatment, then rinsed in PBS and suspended in $500 \mu \mathrm{l}$ binding buffer, and finally incubated with $5 \mu \mathrm{l}$ Annexin $\mathrm{V}$ and $5 \mu \mathrm{l}$ propidium iodide (Nanjing KeyGen Biotech. Inc., Nanjing, China). The stained cells were analyzed by using a BD flow cytometer (FACSCalibur; BD Biosciences, Franklin Lakes, NJ, USA).

Western blot analysis. The cultured primary cardiomyocytes were extracted to harbor protein solution in radioimmunoprecipitation assay lysis buffer (Beyotime Institute of Biotechnology, Nantong, China) after indicated experimental protocols. A BCA Protein Assay Kit (Wuhan Boster Biological Technology Co., Ltd.) was used to detect concentration of the proteins. Extracts containing $20 \mu \mathrm{g}$ of protein were added into each well and subjected to $10 \%$ SDS-PAGE, which were then transferred onto polyvinylidene difluoride membranes (EMD Millipore, Billerica, MA, USA). Subsequently, the membranes were blocked by $5 \% \mathrm{BSA}$ for $60 \mathrm{~min}$ at $37^{\circ} \mathrm{C}$, paralleling the incubation of primary antibodies overnight at $4^{\circ} \mathrm{C}$ with anti-HMGB1 (1:1,000 dilution; \#SAB2701809; Sigma-Aldrich; Merck KGaA), anti-IL-17A (1:800 dilution; \#13838; Cell Signaling Technology, Inc., Danvers, MA, USA), anti- microtubule-associated proteins 1A/1B light chain 3B (LC3) (1:600 dilution; \#SAB1306269; Sigma-Aldrich; Merck KGaA), anti-Beclin-1 (1:800 dilution; \#3738; Cell Signaling Technology, Inc.), anti-Bcl-2 (1:800 dilution; \#15071; Cell Signaling Technology, Inc.) and anti-Bcl-2-associated X protein (Bax) (1:600 dilution; \#2774; Cell Signaling Technology, Inc.). After being washed in Tris buffered saline with Tween 20 for three times (10 min), the membranes were exposed to horseradish peroxidase-conjugated secondary antibody (mouse anti-rat IgG antibody; 1:1,000 dilution; \#bs-0293M; Beijing Bioss Biological Technology Co., Ltd, Beijing, China) at $37^{\circ} \mathrm{C}$ for $2 \mathrm{~h}$. The enhanced chemiluminescence reagent (Pierce; Thermo Fisher Scientific, Inc., Waltham, MA, USA) was used to visualize protein blots. The expression of protein was normalized to $\beta$-actin (mouse anti- $\beta$-actin antibody; 1:500 dilution; \#bsm-33036M; Beijing Bioss Biological Technology Co., Ltd) expression. Image Lab software 3.0 (Bio-Rad Laboratories, Inc., Hercules, CA, USA) was adopted to measure the grey value of each band.

Statistical analysis. Statistical analysis was conducted with SPSS 18.0 software package (SPSS Inc., Chicago, IL, USA). All data are expressed as the mean \pm standard deviation. One-way analysis of variance or the Welch test was used for comparisons among groups, and the Student-Newman-Keuls test or Dunnett's T3 test was used for post-hoc multiple comparisons. $\mathrm{P}<0.05$ was considered to indicate a statistically significant difference.

\section{Results}

HMGB1 and IL-17A aggravate H/R-induced cell injury. As demonstrated in Fig. 1A, cardiomyocytes subjected to hypoxia 

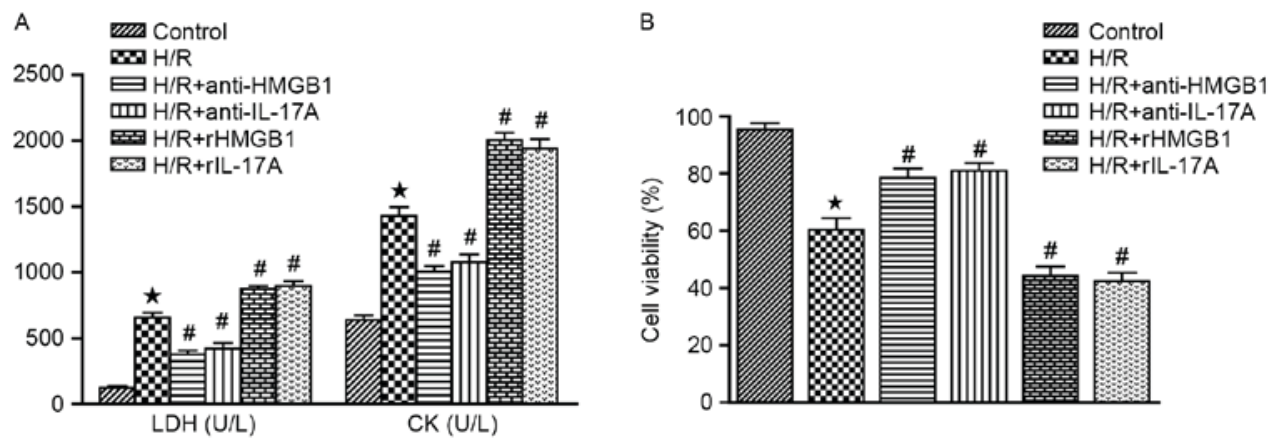

Figure 1. (A) Effects of HMGB1 and IL-17A on the release of LDH and CK. (B) Effects of HMGB1 and IL-17A on the viability of cardiomyocytes. ${ }^{\star} \mathrm{P}<0.05$ vs. Control group; ${ }^{\text {P }}<0.05$ vs. H/R group. HMGB1, high-mobility group box 1 protein; IL, interleukin; LDH, lactate dehydrogenase; CK, creatine kinase; H/R, hypoxia/reoxygenation.

and reoxygenation were able to cause the significant release of LDH and CK, when compared with the control group $(\mathrm{P}<0.05)$. Furthermore, rHMGB1 or rIL-17A increased the levels of LDH and CK, while HMGB1 or IL-17A antibody were able to suppress their release (all $\mathrm{P}<0.05$ vs. $\mathrm{H} / \mathrm{R}$ group).

HMGB1 and IL-17A inhibit the cell viability. The authors assessed cell viability with CCK-8 assays in each groups. The cell viability was markedly inhibited following $\mathrm{H} / \mathrm{R}$ treatment, when compared with the control group $(\mathrm{P}<0.05)$. Interestingly, pretreatment with HMGB1 or IL-17A antibody significantly improved cell viability, while rHMGB1 or rIL-17A could inhibit the cell viability (all $\mathrm{P}<0.05$ vs. H/R group). These findings indicated that HMGB1 or IL-17A could inhibit the cell viability in cardiomyocyte subjected to H/R (Fig. 1B).

HMGB1 and IL-17A aggravate H/R-induced cardiomyocyte apoptosis. Annexin-V FITC/PI staining was used to quantify the pro-apoptotic effects of HMGB1 and IL-17A. The apoptosis rate in $\mathrm{H} / \mathrm{R}$ group was significantly increased $(\mathrm{P}<0.05$ vs. Control). However, pretreatment with HMGB1 or IL-17A antibody could significantly attenuate the elevated H/R-induced cardiomyocyte apoptosis, while rHMGB1 or rIL-17A could aggravate the cardiomyocyte apoptosis (all $\mathrm{P}<0.05$ vs. $\mathrm{H} / \mathrm{R}$ group) (Fig. 2A). In addition, Bcl-2 (anti-apoptotic protein) and Bax (pro-apoptotic protein) expression were also measured by western blotting (Fig. 2B). A significant decrease in Bcl-2 expression and a increase in Bax expression were observed in $\mathrm{H} / \mathrm{R}$ group, when compared with the control group $(\mathrm{P}<0.05)$. Meanwhile, HMGB1 or IL-17A antibody could increase the expression of Bcl-2 and decrease the expression of Bax (both $\mathrm{P}<0.05$ vs. $\mathrm{H} / \mathrm{R}$ group). In contrast, treatment with $\mathrm{rHMGB} 1$ or rIL-17A could attenuate the increased expression of Bcl-2 and decreased the expression of Bax (both $\mathrm{P}<0.05$ vs. H/R group). These results strongly demonstrated that HMGB1 or IL-17A could aggravate H/R-induced cardiomyocyte apoptosis.

HMGBI and IL-17A enhanced the expression levels of autophagy markers in cardiomyocytes subjected to $H / R$. It is well-understood that autophagy is regulated by autophagy-related proteins, such as, LC3-II/LC3-I and Beclin-1 (10). The ratio of LC3-II to LC3-I and the Beclin-1 expression were significantly increased in $\mathrm{H} / \mathrm{R}$ group, when compared with the control group $(\mathrm{P}<0.05)$. The HMGB1 or
IL-17A antibody could markedly decreased the ratio of LC3-II to LC3-I and the Beclin-1 expression when compared to H/R group, while those were even higher in $\mathrm{H} / \mathrm{R}+\mathrm{rHMGB} 1$ group or $\mathrm{H} / \mathrm{R}+\mathrm{rIL}-17 \mathrm{~A}$ group (all $\mathrm{P}<0.05$ ). It can be concluded that the autophagy was upregulated in cardiomyocytes exposed to H/R, and enhanced by HMGB1 or IL-17A prior to H/R (Fig. 3).

$H M G B 1$ regulated the expression of $I L-17 A$. According to the western blotting, both HMGB1 and IL-17A expression in H/R group were markedly increased compared to those in control groups $(\mathrm{P}<0.05)$. Compared with the H/R group, IL-17A expression was significantly decreased in H/R+anti-HMGB1 group, while it was increased in $\mathrm{H} / \mathrm{R}+\mathrm{rHMGB} 1$ group (both $\mathrm{P}<0.05)$. Meanwhile, IL-17A antibody or rIL-17A had no significant effect on HMGB1 expression when compared to $\mathrm{H} / \mathrm{R}$ groups (both $\mathrm{P}>0.05$ ) (Fig. 4).

\section{Discussion}

The present study revealed a crucial role for the HMGB1-IL-17A axis in cardiomyocyte H/R injury. HMGB1 promotes the expression of IL-17A, then aggravates cardiomyocyte apoptosis and autophagy, and finally augments cardiomyocyte $\mathrm{H} / \mathrm{R}$ injury.

Recently, a number of studies have indicated that local inflammation, cell apoptosis and autophagy all contribute to myocardial I/R injury. IL-17A is an inflammatory cytokine with robust effects on different cell types, resulting in inflammatory cytokine production, leukocyte recruitment and creating a link between innate and adaptive immunity (11). It has been reported that IL-17A induces cardiomyocyte apoptosis and promotes ventricular remodeling following myocardial infarction (12). In addition, IL-17A was demonstrated to be significantly increased in a cardiac transplantation model, and could induce cardiomyocyte apoptosis, promote the release of proinflammatory mediators and serve an important role in myocardial I/R injury $(5,13)$. HMGB1, which is a novel pro-inflammatory cytokine actively released by macrophages and monocytes, has been proven to function as an early mediator of inflammation and cell injury during myocardial I/R. In addition, HMGB1 may promote the release of classical early pro-inflammatory cytokines such as tumor necrosis factor- $\alpha$ (TNF- $\alpha$ ) and IL-6. Conversely, HMGB1 A box peptide (a specific HMGB1 antagonist) may inhibit the 

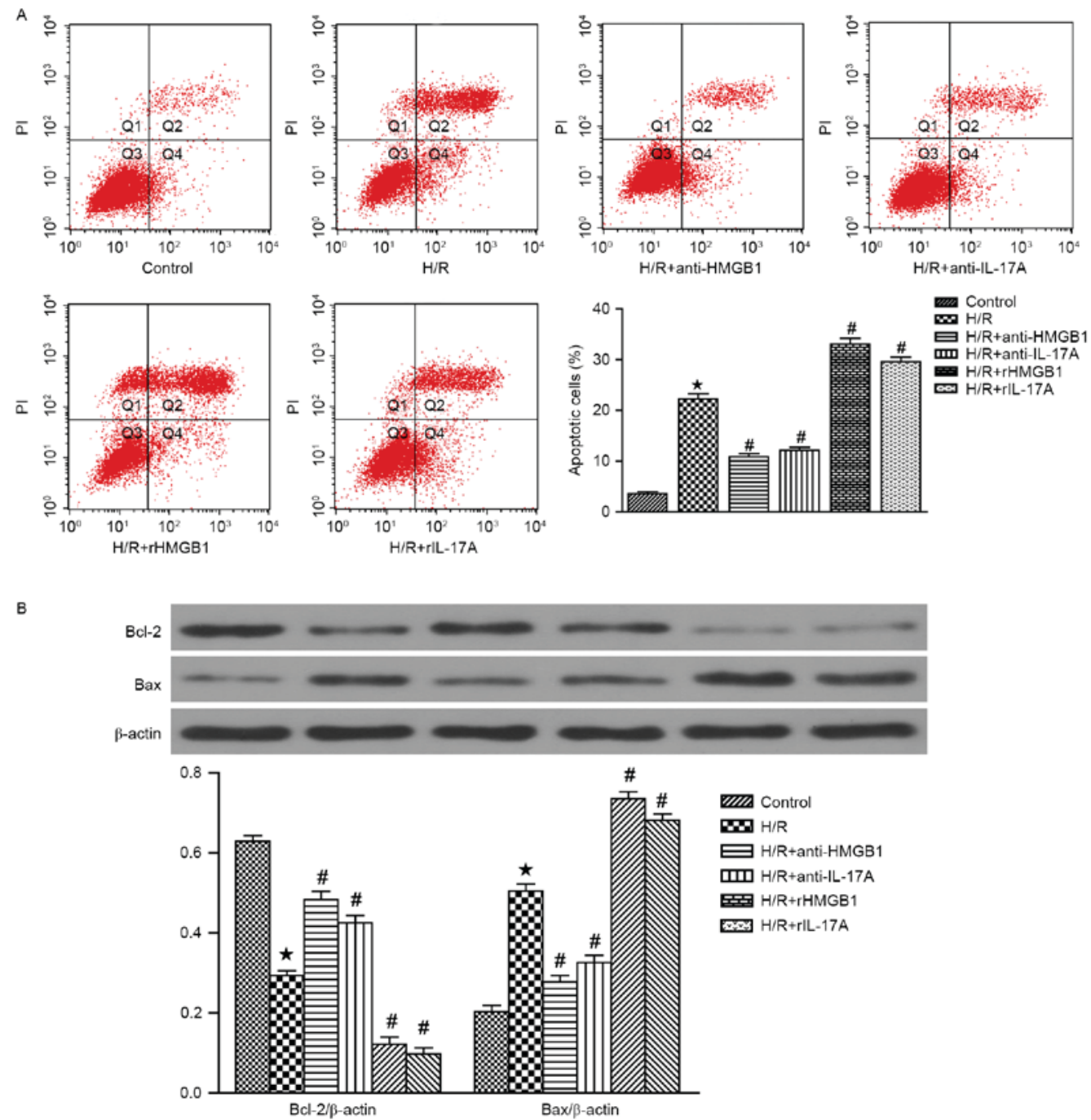

Figure 2. Effects of HMGB1 and IL-17A on the apoptosis of cardiomyocytes. (A) Apoptosis rate was determined by Annexin V-fluorescein isothiocyanate/PI flow cytometry (Q1, Necrotic cells; Q2, Early apoptotic cells; Q3, Viable cells; Q4, Late apoptotic cells). (B) Effects of HMGB1 and IL-17A on the expression of Bcl-2 and Bax in cardiomyocytes. ${ }^{\star} \mathrm{P}<0.05$ vs. Control group; ${ }^{*} \mathrm{P}<0.05$ vs. H/R group. HMGB1, high-mobility group box 1 protein; IL, interleukin; PI, propidium iodide; $\mathrm{H} / \mathrm{R}$, hypoxia/reoxygenation.

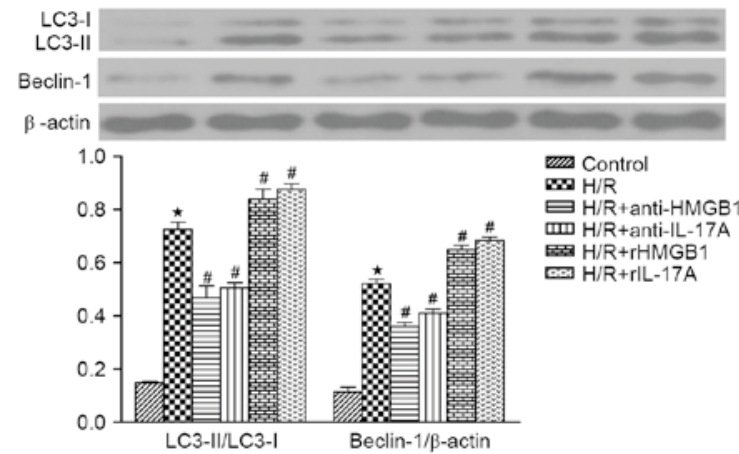

Figure 3. Effects of HMGB1 and IL-17A on the expression of LC3 and Beclin-1 proteins. ${ }^{\star} \mathrm{P}<0.05$ vs. Control group; ${ }^{*} \mathrm{P}<0.05$ vs. H/R group. HMGB1, high-mobility group box 1 protein; IL, interleukin; H/R, hypoxia/reoxygenation.

release of TNF- $\alpha$ and IL- 6 , and reduce myocardial I/R injury. This is due to the mRNA and protein expression of HMGB1 being increased as early as at $30 \mathrm{~min}$ following ischemia, and being significantly increased at $6 \mathrm{~h}$ reperfusion (7). A previous

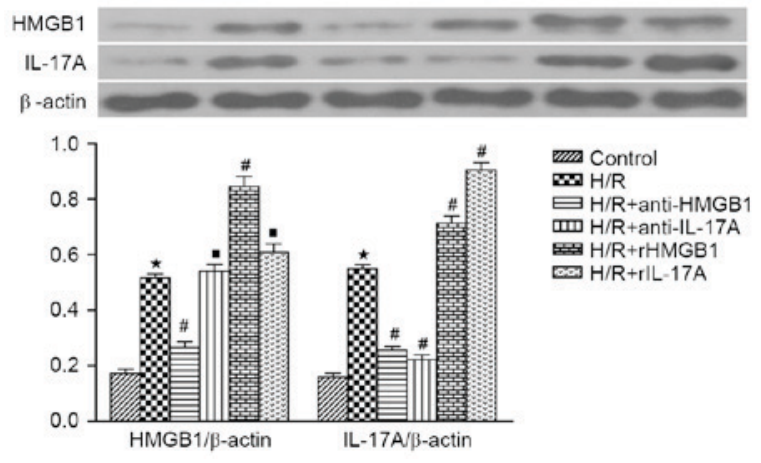

Figure 4. Effects of HMGB1 on the expression of IL-17A in cardiomyocytes. ${ }^{\star} \mathrm{P}<0.05$ vs. Control group; ${ }^{\sharp} \mathrm{P}<0.05$ vs. $\mathrm{H} / \mathrm{R}$ group; ${ }^{\bullet} \mathrm{P}>0.05$ vs. $\mathrm{H} / \mathrm{R}$ group. HMGB1, high-mobility group box 1 protein; IL, interleukin; H/R, hypoxia/reoxygenation.

study of the authors demonstrated that the downregulation of HMGB1 by several drugs, such as minocycline and ethyl pyruvate, can reduce myocardial ischemia/reperfusion injury in rats $(14,15)$. 
A previous study showed that there is an association between HMGB1 and IL-17A (16). Wang, Sun and Tian (16) indicated that both HMGB1 and IL-17A were significantly increased during liver I/R injury, and that HMGB1 may stimulate the production of IL-17A by $\gamma \delta \mathrm{T}$ cells in a TLR4-dependent manner. In the present study, that HMGB1 antibody significantly reduced IL-17A expression and ameliorated cardiomyocyte H/R injury. In contrast, rHMGB1 increased IL-17A expression and markedly increased H/R injury, confirming the crucial role played by HMGB1 in the myocardial I/R. However, the mechanisms underlying the regulation of IL-17A by HMGB1 during myocardial H/R injury require further investigation.

Cardiomyocyte apoptosis is a well-known key cellular event in ischemic hearts (17). In the current study, flow cytometry was performed to examine cardiomyocyte apoptosis. Pretreatment with IL-17A antibody significantly decreased cardiomyocyte apoptosis while rIL-17A have an opposite effect. Apoptosis-related proteins, such as Bcl-2, Bax and caspase-3, play critical roles in apoptosis (18). Overexpression of $\mathrm{Bcl}-2$ in mice attenuates apoptosis and alleviates myocardial IR injury (19). The present results indicated that $\mathrm{H} / \mathrm{R}$ markedly downregulated Bcl-2 and upregulated Bax. Meanwhile, pretreatment with rIL-17A significantly decreased Bcl-2 expression and increased Bax expression, yet the IL-17A antibody increased Bcl-2 expression and decreased Bax expression. These indicated that IL-17A may exert its pro-apoptotic effects through regulating $\mathrm{Bcl}-2$ expression.

Autophagy, another major factor leading to cell death, can be observed in both acute and chronic myocardial ischemia and heart failure $(20,21)$. There is vast evidence that autophagy is upregulated during myocardial ischemia and reperfusion (22). Autophagy may aggravate myocardial I/R injury during the reperfusion phase due to the excessive degradation of essential proteins and organelles (23). A previous study indicated that impaired autophagosome clearance could trigger mitochondrial permeabilization, and then lead to a necrotic mechanism of cell death in cardiac IR injury (24), while attenuating autophagy dysfunction could contribute to cardioprotection (25). These findings are consistent with the present study. The LC3 and Beclin1 proteins are two important markers of autophagosomes, which are upregulated during the reperfusion period and signify ongoing autophagy and cellular damage. The authors' previous study presented that HMGB1 could regulate LC3 and Beclin-1 levels following H/R injury in rat cardiomyocytes (26). In the present study, it was observed that both rHMGB1 and rIL-17A dramatically induced autophagosome formation (as evidenced by the increase of the LC3-II/LC3-I ratio and the upregulation of Beclin 1 expression) in cardiomyocytes subjected to $\mathrm{H} / \mathrm{R}$, while HMGB1 and IL-17A antibodies have an opposite effect. All these results suggested that HMGB1 may promote autophagy through upregulating the expression of IL-17A.

In conclusion, the present current in vitro study contributes to a better understanding of the role of the HMGB1-IL-17A axis in inducing cardiomyocyte apoptosis and promoting autophagy to cell damage following H/R. IL-17A is a key point in controlling HMGB1-induced cardiomyocyte H/R injury. Considering its important role in cardiomyocyte apoptosis and autophagy, a therapeutic approach involving the HMGB1-IL-17A axis and its related mechanisms may constitute a new strategy for myocardial I/R injury.

\section{Acknowledgements}

This study was partially supported by grants from the National Natural Science Foundation of China (grant no. 81370308) and grants from the Natural Science Foundation of Hubei (grant nos. 2013CFB250 and 2015CFB701).

\section{References}

1. Frangogiannis NG, Smith CW and Entman ML: The inflammatory response in myocardial infarction. Cardiovasc Res 53: 31-47, 2002.

2. Gottlieb RA and Engler RL: Apoptosis in myocardial ischemia-reperfusion. Ann N Y Acad Sci 874: 412-426, 1999.

3. Gurusamy N, Lekli I, Gorbunov NV, Gherghiceanu M, Popescu LM and Das DK: Cardioprotection by adaptation to ischaemia augments autophagy in association with BAG-1 protein. J Cell Mol Med 13: 373-387, 2009.

4. Iwakura Y, Nakae S, Saijo S and Ishigame H: The roles of IL-17A in inflammatory immune responses and host defense against pathogens. Immunol Rev 226: 57-79, 2008.

5. Liao YH, Xia N, Zhou SF, Tang TT, Yan XX, Lv BJ, Nie SF, Wang J, Iwakura Y, Xiao H, et al: Interleukin-17A contributes to myocardial ischemia/reperfusion injury by regulating cardiomyocyte apoptosis and neutrophil infiltration. J Am Coll Cardiol 59: 420-429, 2012.

6. Tang D, Kang R, Cheh CW, Livesey KM, Liang X, Schapiro NE, Benschop R, Sparvero LJ, Amoscato AA, Tracey KJ, et al: HMGB1 release and redox regulates autophagy and apoptosis in cancer cells. Oncogene 29: 5299-5310, 2010.

7. Andrassy M, Volz HC, Igwe JC, Funke B, Eichberger SN, Kaya Z, Buss S, Autschbach F, Pleger ST, Lukic IK, et al: High-mobility group box-1 in ischemia- reperfusion injury of the heart. Circulation 117: 3216-3226, 2008.

8. Zhang J, Wu Y, Weng Z, Zhou T, Feng T and Lin Y: Glycyrrhizin protects brain against ischemia-reperfusion injury in mice through HMGB1-TLR4-IL-17A signaling pathway. Brain Res 1582: 176-186, 2014.

9. Hu X, Xu W and Jiang H: HMGB1/IL-17A axis: An important mechanism for myocardial ischemia-reperfusion injury. Int J Cardiol 174: 447-448, 2014.

10. Gurusamy N, Lekli I, Mukherjee S, Ray D, Ahsan MK, Gherghiceanu M, Popescu LM and Das DK: Cardioprotection by resveratrol: A novel mechanism via autophagy involving the mTORC2 pathway. Cardiovasc Res 86: 103-112, 2010.

11. Kolls JK and Lindén A: Interleukin-17 family members and inflammation. Immunity 21: 467-476, 2004.

12. Zhou SF, Yuan J, Liao MY, Xia N, Tang TT, Li JJ, Jiao J, Dong WY, Nie SF, Zhu ZF, et al: IL-17A promotes ventricular remodeling after myocardial infarction. J Mol Med (Berl) 92: 1105-1116, 2014.

13. Zhu H, Li J, Wang S, Liu K, Wang L and Huang L: Hmgb1-TLR4-IL-23-IL17A axis promote ischemia-reperfusion injury in a cardiac transplantation model. Transplantation 95: 1448-1454, 2013

14. Hu X, Zhou X, He B, Xu C, Wu L, Cui B, Wen H, Lu Z and Jiang H: Minocycline protects against myocardial ischemia and reperfusion injury by inhibiting high mobility group box 1 protein in rats. Eur J Pharmacol 638: 84-89, 2010.

15. Hu X, Cui B, Zhou X, Xu C, Lu Z and Jiang H: Ethyl pyruvate reduces myocardial ischemia and reperfusion injury by inhibiting high mobility group box 1 protein in rats. Mol Biol Rep 39: 227-231, 2012.

16. Wang X, Sun R, Wei H and Tian Z: High-mobility group box 1 (HMGB1)-Toll-like receptor (TLR)4-interleukin (IL)-23-IL-17A axis in drug-induced damage-associated lethal hepatitis: Interaction of $\gamma \delta \mathrm{T}$ cells with macrophages. Hepatology 57: 373-384, 2013.

17. Abbate A, Bussani R, Amin MS, Vetrovec GW and Baldi A: Acute myocardial infarction and heart failure: Role of apoptosis. Int J Biochem Cell Biol 38: 1834-1840, 2006.

18. Lopez-Neblina F, Toledo AH and Toledo-Pereyra LH: Molecular biology of apoptosis in ischemia and reperfusion. J Invest Surg 18: 335-350, 2005. 
19. Chen Z, Chua CC, Ho YS, Hamdy RC and Chua BH: Overexpression of $\mathrm{Bcl}-2$ attenuates apoptosis and protects against myocardial I/R injury in transgenic mice. Am J Physio Heart Circ Physiol 280: H2313-H2320, 2001.

20. Costa R, Morrison A, Wang J, Manithody C, Li J and Rezaie AR: Activated protein $\mathrm{C}$ modulates cardiac metabolism and augments autophagy in the ischemic heart. J Thromb Haemost 10 1736-1744, 2012

21. Yan L, Vatner DE, Kim SJ, Ge H, Masurekar M, Massover WH, Yang G, Matsui Y, Sadoshima J and Vatner SF: Autophagy in chronically ischemic myocardium. Proc Natl Acad Sci USA 102: 13807-13812, 2005.

22. Hamacher-Brady A, Brady NR and Gottlieb RA: Enhancing macroautophagy protects against ischemia/reperfusion injury in cardiac myocytes. J Biol Chem 281: 29776-29787, 2006.
23. Takagi H, Matsui Y, Hirotani S, Sakoda H, Asano T and Sadoshima J: AMPK mediates autophagy during myocardial ischemia in vivo. Autophagy 3: 405-407, 2007.

24. Ma X, Liu H, Foyil SR, Godar RJ, Weinheimer CJ, Hill JA and Diwan A: Impaired autophagosome clearance contributes to cardiomyocyte death in ischemia/reperfusion injury. Circulation 125: 3170-3181, 2012.

25. Yao T, Ying X, Zhao Y, Yuan A, He Q, Tong H, Ding S, Liu J, Peng X, Gao E, et al: Vitamin D receptor activation protects against myocardial reperfusion injury through inhibition of apoptosis and modulation of autophagy. Antioxid Redox Signal 22: 633-650, 2015.

26. Xu W, Jiang H, Hu X and Fu W: Effects of high-mobility group box 1 on the expression of Beclin-1 and LC3 proteins following hypoxia and reoxygenation injury in rat cardiomyocytes. Int J Clin Exp Med 7: 5353-5357, 2014. 